\title{
Migrating Intrauterine contraceptive device (IUD) causing hydronephrosis - A case report
}

Bandara KGMW'1, Meegahawatta $\mathrm{AR}^{2}$, Rajapakse $\mathrm{DC}^{3}$

\section{Introduction}

Intrauterine contraceptive device (IUD) is a popular, safe and effective method of contraception and family planning in Sri Lanka ${ }^{1}$. Complications associated with IUD use include uterine perforation. Transmigration of IUD and perforation of abdominal viscera is very rare. We present a case of IUD perforating in to the transverse colon presenting with right sided partial ureteric obstruction causing hydronephrosis.

\section{Case report.}

A 40-year-old female presented with right sided colicky lower abdominal pain associated with fever, dysuria and vomiting for 5 days duration. She also had urinary frequency. Fever accompanied chills with rigors. There was no alteration of bowel habits or bleeding from rectum. On examination, right side renal angle and lower abdominal tenderness was elicited without guarding or rigidity. A pregnancy was excluded by urine dipstick method.

Her investigations showed a neutrophil leukocytosis $\left(17 \times 10^{9} / \mathrm{L}\right)$ with an elevated $C$ reactive protein. Urine analysis revealed 8-10 pus cells per high power field. Her plain x-ray abdomen showed an IUD in an abnormally high position. Subsequent ultrasound examination revealed moderate fullness of right side pelvicalyceal system with dilated upper ureter (right renal pelvis AP $-2.3 \mathrm{~cm}$ ) suggestive of a ureteric stricture or calculus. In addition, the IUD noted in the $x$-ray was not seen within the uterus. No evidence of appendicitis was noted.

Patient was started on empirical antibiotics and a gynecological opinion was obtained. A computerised tomography (CT) of abdomen was suggested for locating the IUD. However, it was decided to precede the CT abdomen with a laparoscopic exploration of the abdominal cavity, taking in to consideration, the clinical findings.

During laparoscopic examination, the uterus was found to be normal in size with no evidence of recent perforation. A mass involving transverse colon and omentum was noted over the right sacroiliac joint where the ureter enters to the pelvis. The mass was carefully dissected

1 Registrar in General Surgery, 2 Medical officer, 3 Consultant General Surgeon District General Hospital Matara

iD https://orcid.org/0000-0002-8998-3925 revealing strings of the IUD leading in to the transverse colon. Due to difficulty in further laparoscopic dissection, the surgery was converted to a mini laparotomy with a small pfannenstiel incision. Further dissection revealed the IUD lodged intraluminaly within the transverse colon with resultant chronic inflammation and mass formation causing mechanical obstruction of right side ureter leading to hydronephrosis. The IUD was extracted, and transverse colon repaired. Post-surgical recovery was uneventful.

\section{Figure 1 - Extracted IUD}

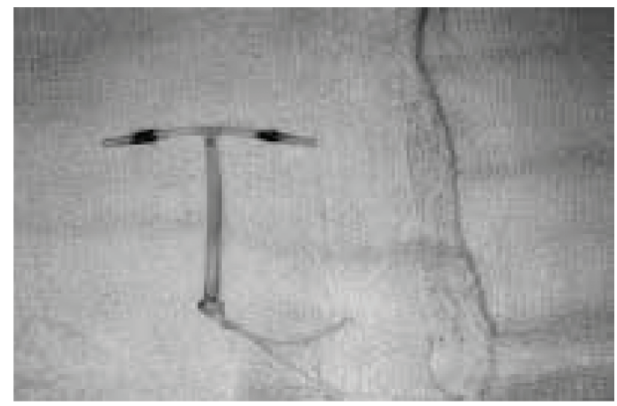

Figure 2 plain $\mathrm{X}$ ray showing the migrated IUD

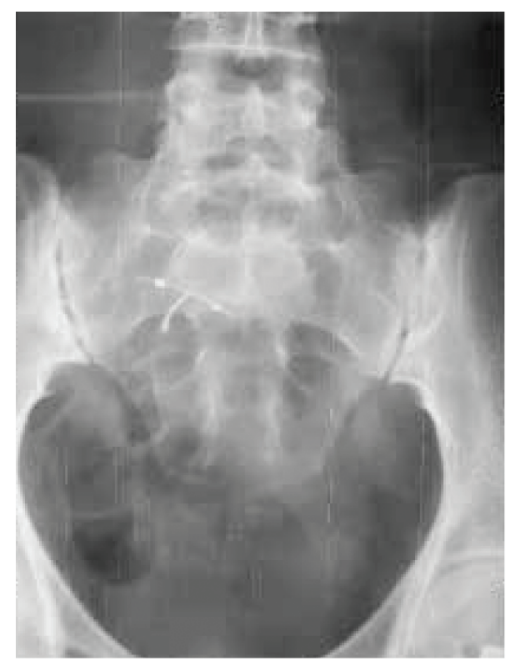

\section{Discussion}

Complications associated with IUD use, while uncommon, include altered menstrual patterns, ectopic pregnancy, infection, ovarian cysts, and rarely uterine perforation ${ }^{2}$. When an IUD is placed, regular periodic self-inspection is advised to ensure accurate positioning of the device. Most perforations are thought to occur during insertion and the risk is highest during the early postpartum period ${ }^{3,4}$. Perforation of abdominal viscera following transmigration of IUD in to the abdominal cavity is extremely rare. Perforation into sigmoid colon, descending colon, appendix and bladder all have been 
reported ${ }^{4}$. Presenting symptoms may wary depending on the location and position of the IUD. Patients will require laparoscopic or open surgical extraction.

On rare occasions IUD's can cause uterine rupture and subsequent intra-abdominal migration. While most cases of rupture occur at the time of insertion. Like in the case above, some patients may present with complications many years after initial placement [4].

In the case above, the patient had neglected and forgotten about the IUD that was placed in her uterus 15 years before. It was eventually recovered from her transverse colon, where it had formed a mass causing ureteric obstruction. It is important to note that, the patient had no alteration of bowel habits or bleeding per-rectum despite the IUD being located intraluminaly in the transverse colon. Regularly checking for the presence of the IUD strings to ensure accurate placement and removing the IUD once the designed usage period has expired are important steps that can help prevent cases such as the one reported here.

\section{References}

1. Sri Lanka \& Family Planning; An overview. Searo.who.int. 2017 cited 16 November 2017. Availablefrom:http://www.searo.who.int/srilanka/ areas/maternal_reproductive_health/srl-familyplanning.pdf?ua=1

2. Kailasam C. Review of the safety, efficacy and patient acceptability of the levonorgestrel-releasing intrauterine system. Patient Preference and Adherence. 2008;2:293.

3. Nagel T. Intrauterine contraceptive devices. Postgraduate Medicine. 1983;73(3):155-164.

4. Mederos R, Humaran L, Minervini D. Surgical removal of an intrauterine device perforating the sigmoid colon: A case report. International Journal of Surgery. 2008;6(6):e60-e62. 